Table S1. Changes in potato sprouts' metabolome in response to Rhizoctonia solani $72 \mathrm{~h}$ post infection. Metabolites with the largest variation in their relative content between control and infected sprouts and essential components of plant defence are displayed. Compound identification (ID) is provided according to the PubChem database (http://pubchem.ncbi.nlm.nih.gov/).

\begin{tabular}{|c|c|c|c|c|c|c|c|}
\hline Metabolites & $\begin{array}{l}\text { Relative content } \\
\text { change in infected } \\
\text { sprouts }(\%)^{a}\end{array}$ & $\begin{array}{l}\text { Relative content } \\
\text { of infected sprouts } \\
\qquad \%)^{a}\end{array}$ & $\begin{array}{l}\text { Analytical } \\
\text { platform }^{b}\end{array}$ & $\begin{array}{l}\text { Molecular } \\
\text { form }\end{array}$ & $\begin{array}{l}\text { Monoisotopic mass } \\
\text { (Da) }\end{array}$ & $P$-values & ID \\
\hline \multicolumn{8}{|l|}{ Alkaloids } \\
\hline \multicolumn{8}{|l|}{ Sesquiterpene alkaloids } \\
\hline Phytuberin & $n / a^{c}$ & 0.20 & $\mathrm{FT}+$ & $\mathrm{C}_{17} \mathrm{H}_{26} \mathrm{O}_{4}$ & 294.1831 & $n / a$ & $10379702, .315114$ \\
\hline Rishitin & $n / a^{c}$ & 0.16 & FT+ & $\mathrm{C}_{14} \mathrm{H}_{22} \mathrm{O}_{2}$ & 222.1619 & $n / a$ & 108064,534260 \\
\hline Solavetivone & $n / a^{c}$ & 1.52 & FT- & $\mathrm{C}_{15} \mathrm{H}_{22} \mathrm{O}$ & 218.1671 & $n / a$ & $\begin{array}{c}10104810,442399 \\
521550,185777 \\
11085279\end{array}$ \\
\hline \multicolumn{8}{|l|}{ Steroidal alkaloids } \\
\hline$\alpha$-Chaconine & -36.82 & 4.78 & FT+ & $\mathrm{C}_{45} \mathrm{H}_{73} \mathrm{NO}_{14}$ & 851.5031 & $<0.0001$ & 104750,442971 \\
\hline$\alpha$-Solanine & -27.81 & 2.02 & FT+ & $\mathrm{C}_{45} \mathrm{H}_{73} \mathrm{NO}_{15}$ & 867.4980 & 0.0022 & $\begin{array}{c}30185,51020424 \\
9549171,262500 \\
6537493\end{array}$ \\
\hline$\beta$-Chaconine & 88.91 & 0.95 & $\mathrm{FT}+$ & $\mathrm{C}_{39} \mathrm{H}_{63} \mathrm{NO}_{10}$ & 705.4452 & $<0.0001$ & 119393 \\
\hline$\beta$-Solanine & $n / a^{c}$ & 0.33 & FT+ & $\mathrm{C}_{39} \mathrm{H}_{63} \mathrm{NO}_{11}$ & 721.4401 & $n / a$ & 45479590 \\
\hline
\end{tabular}




Solanaviol
Solanidine
Solasodenone
Solasodiene
Solasodine

Solasonine

Solaspiralidine

Nortropane alkaloids

Calystegine A3

Calystegine B2

\section{Amino acids}

Protein amino acids 5-Oxo-L-proline

(Pyroglutamic acid)

$L$-asparagine ${ }^{d}$

$L$-glutamine ${ }^{d}$

L-histidine $n / a^{c}$
53.86
$n / a^{c}$
$n / a^{c}$
88.81

0.30

30.59

0.65

1.17

3.89

$n / a^{c}$

0.06

$\mathrm{FT}+\quad \mathrm{C}_{45} \mathrm{H}_{73} \mathrm{NO}_{16} \quad 883.4929$

$-23.45$

40.18

1.07

$\mathrm{GC} \quad \mathrm{C}_{5} \mathrm{H}_{7} \mathrm{NO}_{3}$

129.0426

3.21

$-32.32$

$-57.23$

$-89.74$

FT+ $\quad \mathrm{C}_{27} \mathrm{H}_{43} \mathrm{NO}_{3} \quad 429.3243$

FT+ $\quad \mathrm{C}_{27} \mathrm{H}_{43} \mathrm{NO} \quad 397.3345$

FT+ $\quad \mathrm{C}_{27} \mathrm{H}_{41} \mathrm{NO}_{2} \quad 411.3137$

FT+ $\quad \mathrm{C}_{27} \mathrm{H}_{41} \mathrm{NO} \quad 395.3188$

FT+ $\quad \mathrm{C}_{27} \mathrm{H}_{43} \mathrm{NO}_{2} \quad 413.3294$

FT+

$\mathrm{C}_{27} \mathrm{H}_{41} \mathrm{NO}_{3}$

427.3086

FT+ $\quad \mathrm{C}_{7} \mathrm{H}_{13} \mathrm{NO}_{3} \quad 159.0895$

$\mathrm{FT}+\quad \mathrm{C}_{7} \mathrm{H}_{13} \mathrm{NO}_{4}$

175.0845

0.05

0.62

0.09

GC $\quad \mathrm{C}_{4} \mathrm{H}_{8} \mathrm{~N}_{2} \mathrm{O}_{3} \quad 132.0535$

GC $\quad \mathrm{C}_{5} \mathrm{H}_{10} \mathrm{~N}_{2} \mathrm{O}_{3} \quad 146.0691$

$<0.0001$

6267

0.0005

5961

FT- $\quad \mathrm{C}_{6} \mathrm{H}_{9} \mathrm{~N}_{3} \mathrm{O}_{2} \quad 155.0695 \quad<0.0001$ 


\begin{tabular}{|c|c|c|c|c|c|c|c|}
\hline$L$-isoleucine ${ }^{d}$ & -43.52 & 0.46 & $\mathrm{GC}$ & $\mathrm{C}_{6} \mathrm{H}_{13} \mathrm{NO}_{2}$ & 131.0946 & $<0.0001$ & 6306 \\
\hline$L$-leucine ${ }^{d}$ & -59.25 & 0.15 & $\mathrm{GC}$ & $\mathrm{C}_{6} \mathrm{H}_{13} \mathrm{NO}_{2}$ & 131.0946 & $<0.0001$ & 6106 \\
\hline \multirow[t]{2}{*}{$L$-proline ${ }^{d}$} & -42.87 & 6.53 & GC & $\mathrm{C}_{5} \mathrm{H}_{9} \mathrm{NO}_{2}$ & 115.0633 & $<0.0001$ & 145742 \\
\hline & -79.17 & 0.18 & FT+ & & & 0.0118 & \\
\hline$L$-serine ${ }^{d}$ & -34.43 & 0.68 & GC & $\mathrm{C}_{3} \mathrm{H}_{7} \mathrm{NO}_{3}$ & 105.0426 & $<0.0001$ & 5951 \\
\hline$L$-threonine ${ }^{d}$ & -48.43 & 0.58 & $\mathrm{GC}$ & $\mathrm{C}_{4} \mathrm{H}_{9} \mathrm{NO}_{3}$ & 119.0582 & $<0.0001$ & 6288 \\
\hline \multirow[t]{2}{*}{$L$-valine } & -68.56 & 0.07 & FT+ & $\mathrm{C}_{5} \mathrm{H}_{11} \mathrm{NO}_{2}$ & 117.0790 & $<0.0001$ & 6287 \\
\hline & \multicolumn{7}{|c|}{ Nonprotein amino acids } \\
\hline$\beta$-Alanine ${ }^{d}$ & 20.05 & 0.05 & GC & $\mathrm{C}_{3} \mathrm{H}_{7} \mathrm{NO}_{2}$ & 89.0477 & 0.0246 & 239 \\
\hline 4-aminobutanoic acid $(\mathrm{GABA})^{d}$ & 20.87 & 3.67 & $\mathrm{GC}$ & $\mathrm{C}_{4} \mathrm{H}_{9} \mathrm{NO}_{2}$ & 103.0633 & 0.0205 & 119 \\
\hline Piperidine-2-carboxylic acid & 33.18 & 0.02 & GC & $\mathrm{C}_{6} \mathrm{H}_{11} \mathrm{NO}_{2}$ & 129.0789 & 0.0058 & 849,439227 \\
\hline \multicolumn{8}{|l|}{ (Pipecolic acid) } \\
\hline \multicolumn{8}{|l|}{ CARBOHYDRATES } \\
\hline$D$-Fructose ${ }^{d}$ & -28.01 & 6.41 & GC & $\mathrm{C}_{6} \mathrm{H}_{12} \mathrm{O}_{6}$ & 180.0634 & 0.0216 & 5984 \\
\hline Myo-inositol $^{d}$ & -22.47 & 4.40 & GC & $\mathrm{C}_{6} \mathrm{H}_{12} \mathrm{O}_{6}$ & 180.0634 & 0.0050 & 892 \\
\hline \multicolumn{8}{|l|}{ Carboxylic acids and } \\
\hline \multicolumn{8}{|l|}{ phenolics } \\
\hline 2-Hydroxybutanedioic acid & -71.13 & 0.10 & FT- & $\mathrm{C}_{4} \mathrm{H}_{6} \mathrm{O}_{5}$ & 134.0215 & 0.0039 & 222656 \\
\hline \multicolumn{8}{|l|}{ (Malic acid) } \\
\hline 2-Hydroxyundecanoic acid & $\begin{array}{l}-21.52 \\
-62.67\end{array}$ & $\begin{array}{l}6.64 \\
0.36\end{array}$ & $\begin{array}{l}\text { GC } \\
\text { GC }\end{array}$ & $\mathrm{C}_{11} \mathrm{H}_{22} \mathrm{O}_{3}$ & 202.1569 & $<0.0001$ & 5282899 \\
\hline 2-Thiobarbituric acid & 20.9 & 5.96 & GC & $\mathrm{C}_{4} \mathrm{H}_{4} \mathrm{~N}_{2} \mathrm{O}_{2} \mathrm{~S}$ & 143.9993 & 0.0490 & 2723628 \\
\hline Citramalic acid & 92.75 & 0.25 & FT- & $\mathrm{C}_{5} \mathrm{H}_{8} \mathrm{O}_{5}$ & 148.0372 & $<0.0001$ & 1081 \\
\hline Chlorogenic acid & -95.03 & 0.01 & FT- & $\mathrm{C}_{16} \mathrm{H}_{18} \mathrm{O}_{9}$ & 354.0950 & $<0.0001$ & 1794427 \\
\hline$D$-galacturonic acid & -21.58 & 0.06 & GC & $\mathrm{C}_{6} \mathrm{H}_{10} \mathrm{O}_{7}$ & 194.0427 & 0.0356 & 3627 \\
\hline$D$-gluconic acid & 56.35 & 2.69 & $\mathrm{GC}$ & $\mathrm{C}_{6} \mathrm{H}_{12} \mathrm{O}_{7}$ & 196.0583 & 0.0209 & 10690 \\
\hline
\end{tabular}


$D$-glucuronic acid

Ferulic acid

$N$-feruloylputrescine

$\mathrm{N}$-feruloyltyramine

Nonanedioic acid ${ }^{d}$ (Azelaic acid)

Pentanedioic acid (Glutaric acid)

Succinic acid $^{d}$

Fatty acids and peroxides

(Z)-Octadec-9-enoic acid(Oleate)

$-24.61$

$-31.40$

Colneleic acid

Colnelenic acid

Hydroperoxyoctadecatrienoic

acid (Hydroperoxylinolenic acid)

Hydroxyoctadecadienoic acid

(Hydroxylinoleic acid)

Octadecadienoic acid (Linolate)

Trihydroxyoctadecanoic acid

(Trihydroxystearic acid)

\section{Several groups}

$\alpha$-Tocotrienol

58.95

2.39

69.82
FT-

$\mathrm{C}_{18} \mathrm{H}_{34} \mathrm{O}_{2}$

282.2558

GC

FT-

FT-

$\mathrm{C}_{18} \mathrm{H}_{30} \mathrm{O}_{3}$

294.2194

FT-

$\mathrm{C}_{18} \mathrm{H}_{28} \mathrm{O}_{3}$

292.2038

$\mathrm{C}_{18} \mathrm{H}_{30} \mathrm{O}_{4} \quad 310.2144$

FT+

$$
\mathrm{C}_{18} \mathrm{H}_{32} \mathrm{O}_{3}
$$

296.2351

0.0487

0.0172

0.0009

0.0451

0.0205

$<0.0001$

$<0.0001$

1110

445858, 1548883

5281796

5280537

2266

743

0.0487

0.0057

0.0012

0.0457

0.0378

0.0346

FT-

FT-

$\mathrm{C}_{18} \mathrm{H}_{32} \mathrm{O}_{2}$

280.2402

0.0001

0.0167

FT-

$\mathrm{C}_{18} \mathrm{H}_{36} \mathrm{O}_{5}$

332.2563

0.0015
445639

6441681

6441679

5497123, 6440263, 5282866

5282948, 6443013, 5282945, 5312833

5281117,5312830

6433819, 6440705

5280450

147011 
(Indolebutyric acid)

${ }^{a}$ Values refer to the relative (\%) peak area or amplitude of total peak area or amplitude and represent the mean of eight biological replications

${ }^{b}$ FT+; Fourier transform-ion cyclotron resonance-mass spectrometry (FT-ICR/MS)-positive mode, FT-; FT-ICR/MS-negative mode, GC; gas chromatography MS

${ }^{c}$ not detected in controls

${ }^{d}$ Metabolites that were definitely identified based on matching their mass spectra and retention times to those of the authentic chemical standards analyzed on the same system with the same analytical method. 